\title{
Yet another version of Mumford's theorem
}

\author{
Robert Laterveer
}

\begin{abstract}
The aim of this note is to provide a variant statement of Mumford's theorem. This variant states that for a general variety, all Chow groups are "as large as possible", in the sense that they cannot be supported on a divisor.
\end{abstract}

Mathematics Subject Classification (2010). Primary 14C15; Secondary $14 \mathrm{C} 25$.

Keywords. Algebraic cycles, Chow groups.

\section{Introduction}

Mumford's theorem $[9$ asserts that for a general variety over $\mathbb{C}$, the Chow group of 0 -cycles is very large. One version of Mumford's theorem states that for a variety $X$ with geometric genus $p_{g}(X)$ non-zero, the Chow group $A^{n} X$ is not supported on any closed subvariety:

Theorem 1.1. (Bloch-Srinivas [4]) Let $X$ be a smooth projective variety of dimension $n$, and suppose $A^{n} X_{\mathbb{Q}}$ is supported on a divisor. Then $p_{g}(X)=0$.

Since the seminal paper [4, a plethora of variant statements and generalizations have seen the day (cf. [17, Chapter 3] for a recent and comprehensive overview of the field). The modest aim of this short expository note is to provide yet one more variant statement, showing that for a general variety, all Chow groups are very large. The price to pay for starting out not with 0 -cycles but with cycles of arbitrary codimension $i$ is that we need to assume the standard Lefschetz conjecture $B(X)$. Here is the main result of this note:

Theorem 1.2. Let $X$ be a smooth projective variety, and suppose $B(X)$ is true. Suppose there is an $i$ such that the Chow group $A^{i}(X)_{\mathbb{Q}}$ is supported on a divisor. Then the cohomology group $H^{i}(X, \mathbb{Q})$ is supported on a divisor.

This can be used to provide instances of varieties for which all Chow groups are very large: 
Corollary 1.3. Let $X$ be an abelian variety. Then no Chow group $A^{i}(X)_{\mathbb{Q}}$ is supported on a divisor.

More examples of this type are given below (Corollary [3.2); the same statement holds for any variety for which one knows $B(X)$ and whose Hodge diamond is of maximal width. This is not surprising, and probably known to experts, yet we couldn't find a reference. Closely related results appear in work of Lewis [7, 8] and Schoen [10, yet their statements (as well as the proofs) are slightly different from ours 1

The present note was written while preparing for the Strasbourg "groupe de travail" based on the book [17. I'd like to thank the participants of this groupe de travail for a very pleasant and stimulating atmosphere.

Convention. In this note, the word variety refers to a smooth projective algebraic variety over $\mathbb{C}$.

\section{The Lefschetz standard conjecture}

Let $X$ be a smooth projective variety of dimension $n$, and $h \in H^{2}(X, \mathbb{Q})$ the class of an ample line bundle. The hard Lefschetz theorem asserts that the map

$$
L^{n-i}: H^{i}(X, \mathbb{Q}) \rightarrow H^{2 n-i}(X, \mathbb{Q})
$$

obtained by cupping with $h^{n-i}$ is an isomorphism, for any $i<n$. One of the standard conjectures asserts that the inverse isomorphism is algebraic.

Definition 2.1. For a given $i<n$, we say that $B(X, i)$ holds if for all ample $h$ the isomorphism

$$
\left(L^{n-i}\right)^{-1}: H^{2 n-i}(X, \mathbb{Q}) \stackrel{\cong}{\rightarrow} H^{i}(X, \mathbb{Q})
$$

is induced by a correspondence.

Definition 2.2. (Lefschetz standard conjecture $B(X)$ ) Following convention, we say that $B(X)$ holds if $B(X, i)$ holds for all $i=0, \ldots, n-1$.

For later use, we recall the notion of geometric coniveau:

Definition 2.3. (geometric coniveau) The geometric coniveau filtration on cohomology is defined as

$$
N^{j} H^{i}(X, \mathbb{Q})=\sum_{Z \subset X} \operatorname{Im}\left(H_{Z}^{i}(X, \mathbb{Q}) \rightarrow H^{i}(X, \mathbb{Q})\right),
$$

where $Z$ runs through all subschemes of $X$ of codimension $\geq j$.

We define a pool of examples for which $B(X)$ is known to hold:

Definition 2.4. Let $\mathcal{B}$ be the class of varieties defined by the following rules:

\footnotetext{
${ }^{1}$ Both Lewis and Schoen suppose the generalized Hodge conjecture holds true, rather than "only" $B(X)$. Also, both work with the notion of "representable Chow group", rather than with the notion of "Chow group supported on a divisor".
} 
(1) The following varieties are in $\mathcal{B}$ :

(i) Curves and surfaces;

(ii) Threefolds not of general type (i.e. having Kodaira dimension $<3$ )

(iii) Abelian varieties;

(iv) $n$-dimensional varieties $X$ which have $A_{i}(X)_{\mathbb{Q}}$ supported on a subvariety of dimension $i+2$ for all $i \leq \frac{n-3}{2}$;

(v) $n$-dimensional varieties $X$ which have $H_{i}(X, \mathbb{Q})=N^{\left\llcorner\frac{i}{2}\right\lrcorner} H_{i}(X, \mathbb{Q})$ for all $i>n$.

(2) $\mathcal{B}$ is closed under taking products, and under taking smooth hyperplane sections.

(3) $\mathcal{B}$ is closed under blow-up, i.e. if $\widetilde{X}$ is the blow-up of $X$ with center $Y$, then $\widetilde{X}$ is in $\mathcal{B}$ if and only if $X$ and $Y$ are in $\mathcal{B}$.

Proposition 2.5. For $X$ in $\mathcal{B}$, the Lefschetz standard conjecture $B(X)$ is true.

Proof. For curves, surfaces and abelian varieties, this is proven by Kleiman 5 , $\S 2$ Appendix]. The case of threefolds not of general type was proven by Tankeev [12]. Case (iv) is [14, Theorem 7.1]. Case (v) follows from [15, Theorem $4.2]$.

The fact that products and hyperplane sections preserve the truth of $B(X)$ is well-known [6]. The statement for blow-ups is proven in [11].

Remark 2.6. Point (iv) of Definition 2.4 implies that rationally connected fourfolds are in $\mathcal{B}$. It also implies that all linear varieties (as defined in [13]) are in $\mathcal{B}$; this class includes toric varieties and spherical varieties. Point (v) implies that every threefold with $h^{0,2}=0$ is in $\mathcal{B}$.

Remark 2.7. Point (3) of Definition 2.4 implies the following: if $X$ is a variety of dimension $\leq 4$, and $X$ is birational to a variety in $\mathcal{B}$, then $X \in \mathcal{B}$.

\section{Main result}

Theorem 3.1. Let $X$ be a smooth projective variety over $\mathbb{C}$. Suppose there is an $i$ such that the Chow group $A^{i}(X)_{\mathbb{Q}}$ is supported on a divisor, and that $B(X, j)$ is true for $j \leq i$. Then the cohomology group $H^{i}(X, \mathbb{Q})$ is supported on a divisor.

This is useful in showing the following: for a general variety, all the Chow groups are as large as possible. Here a "general variety" means a variety having Hodge diamond of maximal width, and "large Chow group" means not supported on a subvariety.

Corollary 3.2. Let $X$ be a variety in $\mathcal{B}$. Suppose for all $i=1, \ldots, n$ the Hodge numbers $h^{i, 0}(X)$ are $\neq 0$. Then there is no Chow group $A^{i}(X)_{\mathbb{Q}}$ supported on a divisor. 
Proof. (of Corollary 3.2) This is immediate from Theorem 3.1, plus the fact that $H^{i}(X, \mathbb{C})$ being supported on a divisor implies (by functoriality of the Hodge filtration) that $H^{i, 0} X$ is 0 .

By way of example, we present two explicit instances of Corollary 3.2 .

Corollary 3.3. Let $X$ be an abelian variety. Then no Chow group $A^{i}(X)_{\mathbb{Q}}$ is supported on a divisor.

Corollary 3.4. Let $L$ be any variety in $\mathcal{B}$ of dimension $m$. Let $C_{1}, \ldots, C_{r}$ be non-rational curves. Let

$$
X \subset\left(L \times C_{1} \times \cdots \times C_{r}\right)
$$

be a complete intersection of codimension $\geq m$. Then no Chow group $A^{i}(X)_{\mathbb{Q}}$ is supported on a divisor.

Proof. (of Theorem 3.1) For $i$ equal to $n=\operatorname{dim} X$, this is Mumford's theorem. We will now suppose $i<n$.

The fact that $B(X, j)$ is true for $j \leq i$ implies [6, Theorem 4-1] that the Künneth component of the diagonal

$$
\pi_{i} \in \operatorname{Im}\left(H^{2 n-i}(X, \mathbb{Q}) \otimes H^{i}(X, \mathbb{Q}) \rightarrow H^{2 n}(X \times X, \mathbb{Q})\right)
$$

is algebraic. Let $h \in H^{2}(X, \mathbb{Q})$ be the class of a very ample line bundle, and let $Y \subset X$ be a general complete intersection of dimension $i$ with class $[Y]=h^{n-i} \in H^{2 n-2 i}(X, \mathbb{Q})$. The weak Lefschetz theorem gives a surjection

$$
H^{i}(Y, \mathbb{Q}) \rightarrow H^{2 n-i}(X, \mathbb{Q}),
$$

implying that actually $\pi_{i}$ is in the image of the composite map

$$
\pi_{i} \in \operatorname{Im}\left(H^{i}(Y, \mathbb{Q}) \otimes H^{i}(X, \mathbb{Q}) \rightarrow H^{2 i}(Y \times X, \mathbb{Q}) \rightarrow H^{2 n}(X \times X, \mathbb{Q})\right) .
$$

Using Lemma 3.5 proved below, we can find an algebraic class

$$
\pi_{i}^{\prime} \in H^{2 i}(Y \times X, \mathbb{Q})
$$

representing $\pi_{i}$ (i.e. the push-forward of $\pi_{i}^{\prime}$ equals $\pi_{i}$ in $H^{2 n}(X \times X, \mathbb{Q})$ ). We can thus lift $\pi_{i}^{\prime}$ to the Chow group $A^{i}(Y \times X)_{\mathbb{Q}}$ and, under the assumption of Theorem 3.1. we can apply the Bloch-Srinivas argument [4] (in the form of Proposition 3.7 below) to get a decomposition

$$
\pi_{i}^{\prime}=\Gamma_{1}+\Gamma_{2} \in A^{i}(Y \times X)_{\mathbb{Q}},
$$

where $\Gamma_{1}$ (resp. $\Gamma_{2}$ ) is supported on $Y^{\prime} \times X$ where $Y^{\prime} \subset Y$ is a divisor (resp. supported on $Y \times D$, where $D \subset X$ is a divisor). Going back to cohomology, this induces a decomposition of the Künneth component

$$
\pi_{i}=\Gamma_{1}+\Gamma_{2} \in H^{2 n}(X \times X, \mathbb{Q}),
$$

with $\Gamma_{1}, \Gamma_{2}$ as above. We now consider the action of the correspondence $\pi_{i}$ on $H^{i}(X, \mathbb{Q})$ :

$$
\mathrm{id}=\left(\pi_{i}\right)_{*}=\left(\Gamma_{1}\right)_{*}+\left(\Gamma_{2}\right)_{*}: H^{i}(X, \mathbb{Q}) \rightarrow H^{i}(X, \mathbb{Q}) .
$$


Because $\Gamma_{2}$ is supported on $Y \times D$, we obviously have

$$
\left(\Gamma_{2}\right)_{*} H^{i}(X, \mathbb{Q}) \subset H_{D}^{i}(X, \mathbb{Q}) \subset N^{1} H^{i}(X, \mathbb{Q}) .
$$

As for $\Gamma_{1}$, we have that $\Gamma_{1}$ is supported on $Y^{\prime} \times X$. This means that the action of $\Gamma_{1}$ factors over $H^{i}\left(\widetilde{Y^{\prime}}, \mathbb{Q}\right)$, where $\widetilde{Y^{\prime}} \rightarrow Y^{\prime}$ is a resolution of singularities. Now, $H^{i}\left(\widetilde{Y^{\prime}}, \mathbb{Q}\right)$ is supported on a divisor $Y^{\prime \prime}$ (weak Lefschetz for the $(i-1)$ dimensional variety $\left.\widetilde{Y^{\prime}}\right)$. It follows that the image of $H^{i}\left(\widetilde{Y^{\prime}}, \mathbb{Q}\right)$ in $H^{i}(X, \mathbb{Q})$ via the correspondence $\Gamma_{1}$ is supported on the divisor

$$
p r_{2}\left(\operatorname{Supp}\left(\Gamma_{1}\right) \cap\left(p r_{1}\right)^{-1}\left(Y^{\prime \prime}\right)\right) \subset X,
$$

and hence

$$
\left(\Gamma_{1}\right)_{*} H^{i}(X, \mathbb{Q}) \subset N^{1} H^{i}(X, \mathbb{Q})
$$

Lemma 3.5. Let $X$ be a variety for which $B(X, i)$ holds. Let $Y \subset X$ a smooth complete intersection of dimension $i$ and with $[Y]=h^{n-i} \in H^{2 n-2 i}(X, \mathbb{Q})$, for some $h \in H^{2}(X, \mathbb{Q})$ the class of an ample line bundle. Suppose a class

$$
c \in \operatorname{Im}\left(H^{i}(Y, \mathbb{Q}) \otimes H^{i}(X, \mathbb{Q}) \rightarrow H^{2 i}(Y \times X, \mathbb{Q}) \rightarrow H^{2 n}(X \times X, \mathbb{Q})\right)
$$

is algebraic. Then there exists an algebraic class

$$
c^{\prime} \in H^{2 i}(Y \times X, \mathbb{Q})
$$

representing $c$.

Proof. Let $\gamma$ denote the correspondence inducing the isomorphism

$$
H^{2 n-i}(X, \mathbb{Q}) \stackrel{\cong}{\rightarrow} H^{i}(X, \mathbb{Q})
$$

inverse to the cup-product with $h^{n-i}$, and let $\Delta \in H^{2 n}(X \times X, \mathbb{Q})$ denote the class of the diagonal. Then $\gamma \times \Delta$ is a correspondence that acts $(\gamma \times \Delta)_{*}: \operatorname{Im}\left(H^{2 n-i} X \otimes H^{i} X \rightarrow H^{2 n}(X \times X)\right) \rightarrow \operatorname{Im}\left(H^{i} X \otimes H^{i} X \rightarrow H^{2 i}(X \times X)\right)$.

It follows that

$$
c^{\prime \prime}:=(\gamma \times \Delta)_{*} c \in H^{2 i}(X \times X)
$$

is algebraic. But then, denoting $j: Y \rightarrow X$ the inclusion, the restriction

$$
c^{\prime}:=(j \times \mathrm{id})^{*}\left(c^{\prime \prime}\right) \in H^{2 i}(Y \times X)
$$

is algebraic as well. But the composition $j_{*} j^{*}$ is cup-product with $h^{n-i}$ on cohomology, so that

$$
j_{*} j^{*} \gamma_{*}=\mathrm{id}: H^{2 n-i}(X, \mathbb{Q}) \rightarrow H^{2 n-i}(X, \mathbb{Q}) .
$$

This implies that

$$
\begin{aligned}
(j \times \mathrm{id})_{*}\left(c^{\prime}\right) & =(j \times \mathrm{id})_{*}(j \times \mathrm{id})^{*}(\gamma \times \mathrm{id})_{*} c \\
& =\left(j_{*} j^{*} \gamma_{*} \times \mathrm{id}\right) c \\
& =c \quad \in H^{2 n}(X \times X, \mathbb{Q}) .
\end{aligned}
$$


Remark 3.6. Lemma 3.5 is a particular case of Voisin's standard conjecture 17. Conjecture 2.29], [16. That it can be proven here is because we suppose $B(X, i)$; this is a particular instance of the fact that the Lefschetz standard conjecture implies Voisin's standard conjecture [17, Proposition 2.32].

Proposition 3.7. (Bloch-Srinivas-style) Let $X$ be a smooth projective variety of dimension $n$. Suppose that for some $i=1, \ldots, n$, the Chow group $A^{i}(X)_{\mathbb{Q}}$ is supported on a subvariety $Z \subset X$. Then for any variety $Y$, and any cycle $\pi \in A^{i}(X \times Y)_{\mathbb{Q}}$, there is a decomposition

$$
\pi=\Gamma_{1}+\Gamma_{2} \in A^{i}(X \times Y)_{\mathbb{Q}},
$$

where $\Gamma_{1}$ is supported on $Z \times Y$, and $\Gamma_{2}$ is supported on $X \times Y^{\prime}$, where $Y^{\prime}$ is a divisor on $Y$.

Proof. We may suppose everything $(X, Z, Y$ and $\pi)$ is defined over a field $k \subset \mathbb{C}$ which is finitely generated over its prime subfield. Let $k(Y)$ denote the function field of $Y$. Since $k(Y) \subset \mathbb{C}$, we have a map

$$
A^{i}\left(X_{k(Y)}\right)_{\mathbb{Q}} \rightarrow A^{i}\left(X_{\mathbb{C}}\right)_{\mathbb{Q}}
$$

which is injective [3, Appendix to Lecture 1]. Hence the hypothesis on $A^{i}\left(X_{\mathbb{C}}\right)_{\mathbb{Q}}$ implies that $A^{i}\left(X_{k(Y)}\right)_{\mathbb{Q}}$ is supported on the subvariety $Z$. On the other hand,

$$
A^{i}\left(X_{k(Y)}\right)_{\mathbb{Q}}=\lim _{\longrightarrow} A^{i}(X \times U)_{\mathbb{Q}},
$$

where the limit is taken over opens $U \subset Y$ [3, Appendix to lecture 1]. Given the cycle $\pi$, we can thus find an open $j: U_{0} \subset Y$ such that the restriction $j^{*} \pi$ equals some cycle $\gamma$ on $Z \times U_{0}$ :

$$
j^{*} \pi=\gamma \in A^{i}\left(X \times U_{0}\right)_{\mathbb{Q}} .
$$

Now defining $\Gamma_{1} \in A^{i}(X \times Y)_{\mathbb{Q}}$ to be any cycle supported on $Z \times Y$ that restricts to $\gamma$, this means we have

$$
j^{*}\left(\pi-\Gamma_{1}\right)=0 \in A^{i}\left(X \times U_{0}\right)_{\mathbb{Q}} ;
$$

i.e. the difference $\Gamma_{2}:=\pi-\Gamma_{1}$ is supported on $X \times\left(Y \backslash U_{0}\right)$.

Remark 3.8. The Bloch-Srinivas style Proposition 3.7 can also be deduced as a special case of Voisin's presentation [17, Theorem 3.1] of the decomposition principle.

Remark 3.9. It is established in [2] that correspondences act on gradeds of the geometric coniveau filtration. This gives another way of concluding, at the end of the proof of Theorem 3.1, that

$$
\left(\Gamma_{1}\right)_{*} H^{i}(X, \mathbb{Q}) \subset N^{1} H^{i}(X, \mathbb{Q}) .
$$

The more direct and explicit argument presented above was suggested by the anonymous referee, to whom we are grateful. 


\section{References}

[1] D. Arapura, Motivation for Hodge cycles, Advances in Math. (2006),

[2] D. Arapura and S.-J. Kang, Functoriality of the coniveau filtration, Canad. Math. Bull. (2007),

[3] S. Bloch, Lectures on algebraic cycles, Duke Univ. Math. Series, Vol. IV,

[4] S. Bloch and V. Srinivas, Remarks on correspondences and algebraic cycles, American Journal of Mathematics Vol. 105, No 5 (1983), 1235-1253,

[5] S. Kleiman, Algebraic cycles and the Weil conjectures, in: Dix exposés sur la cohomologie des schémas, North-Holland Amsterdam, 1968, 359-386,

[6] S. Kleiman, The standard conjectures, in: Motives (U. Jannsen et alii, eds.), Proceedings of Symposia in Pure Mathematics Vol. 55 (1994), Part 1,

[7] J. Lewis, Towards a generalization of Mumford's theorem, J. Math. Kyoto Univ. 29 (1989), 267-272,

[8] J. Lewis, A generalization of Mumford's theorem, II, Illinois Journal of Mathematics Vol. 39 No 2 (1995), 288-304,

[9] D. Mumford, Rational equivalence of 0-cycles on surfaces, J. Math. Kyoto Univ. Vol. 9 No 2 (1969), 195-204,

[10] C. Schoen, On Hodge structures and non-representability of Chow groups, Comp. Math. 88 (1993), 285-316,

[11] S. Tankeev, Monoidal transformations and conjectures on algebraic cycles, Izvestiya Math. 71 (2007), no. 3, 629-655,

[12] S. Tankeev, On the standard conjecture of Lefschetz type for complex projective threefolds. II, Izvestiya Math. 75:5 (2011), 1047-1062,

[13] B. Totaro, Chow groups, Chow cohomology, and linear varieties, Forum of Mathematics, Sigma (2014), vol. 1, e1,

[14] C. Vial, Algebraic cycles and fibrations, Documenta Math. 18 (2013), 15211553 ,

[15] C. Vial, Projectors on the intermediate algebraic Jacobians, New York J. Math. 19 (2013), 793-822,

[16] C. Voisin, The generalized Hodge and Bloch conjectures are equivalent for general complete intersections, Annales scientifiques de l'ENS 46, fascicule 3 (2013), 449-475,

[17] C. Voisin, Chow Rings, Decomposition of the Diagonal, and the Topology of Families, Princeton University Press, Princeton and Oxford,

Robert Laterveer

CNRS, Institut de Recherche Mathématiques Avancées

Université de Strasbourg

7 rue René Descartes

F-67084 Strasbourg Cedex

France

e-mail: laterv@math.unistra.fr 